\section{THU0668 RELIABILITY OF PATIENT GLOBAL ASSESSMENT IN RHEUMATOID ARTHRITIS PATIENTS}

G. Kageyama ${ }^{1}$, A. Onishi ${ }^{2}$, Y. Ueda ${ }^{2}$, J. Saegusa ${ }^{2}$, A. Morinobu ${ }^{2} .{ }^{1}$ Department of Rheumatology, Hyogo Prefectural Amagasaki General Medical Center, Amagasaki; ${ }^{2}$ Department of Rheumatology, Kobe University Hospital, Kobe, Japan

Background: Patient's global assessment (PtGA) is one of the most widely used patient reported outcomes in rheumatoid arthritis (RA) that reflects both disease activity and other factors. PtGA is onymous and obtained at hospital, which may cause a conscious or unconscious bias, whereas PtGA obtained anonymously may be free from any bias. The credibility of PtGA to report RA patient outcomes has been usually investigated by assessing test-retest reliability. There has been no study comparing routine PtGA and PtGA where patients answered anonymously.

Objectives: The aim of this study was to compare routinely obtained in-hospital PtGA before clinical examination with those answered anonymously at home. Additionally, physician's global assessment (PhyGA) was compared with routine PtGA and anonymized PtGA

Methods: We asked RA patients $(n=389)$ to answer and mail the PtGA test anonymously, Clinical data regarding disease duration, stage, class, swollen joint counts, tender joint counts, pain visual analog scale (VAS), PhyGA, Health Assessment Questionnaire (HAQ), EuroQOL five dimensions questionnaire (EQ5D), Kessler 6 scale (anxiety/ depression), treatment data, laboratory data, and socioeconomic factors were collected simultaneously. We compared the PtGA that is routinely surveyed at hospital before clinical examination with those surveyed anonymously at home. We calculated a discrepancy score by subtracting anonymized PtGA from routine in-hospital PtGA. We defined (1) positive discrepancy when routine PtGA was over-rated relative to the anonymized PtGA; (2) negative discrepancy when routine in-hospital PtGA was under-rated relative to the anonymized PtGA.

Results: The anonymized PtGA score was higher than routinely evaluated in-hospital PtGA $(p<0.0001)$. The anonymized PtGA poorly correlated with routine in-hospital PtGA $(r=0.426, p<0.0001)$. We compared patients who had discordance between in-hospital PtGA and anonymized PtGA. We used 3 models in which the discordance between both PtGAs was set at $10 \mathrm{~mm}, 20 \mathrm{~mm}$, or 30 $\mathrm{mm}$. If we adopted $30 \mathrm{~mm}$ as discordance, the pain scale remained to be a risk factor of positive discrepancy (higher in-hospital PtGA than anonymized PtGA). If we adopted $20 \mathrm{~mm}$ or $10 \mathrm{~mm}$ as discordance, the pain scale remained to be a risk factor of positive discrepancy and remaining low quality of life (QOL) negative discrepancy (lower in-hospital PtGA than anonymized PtGA) after multivariate analysis. The discordance between PhyGA and routine PtGA are associated with high pain VAS. The discordance between PhyGA and anonymized PtGA is associated with tender joint counts, swollen joint counts, and low QOL.

\begin{tabular}{cccc}
\hline \multirow{2}{*}{ Discordance Model } & \multicolumn{3}{c}{ Difference between in-hospital PtGA and anonymized PtGA } \\
\cline { 2 - 4 } & $30 \mathrm{~mm}$ & $20 \mathrm{~mm}$ & $10 \mathrm{~mm}$ \\
\hline Household income & $-0.074 \pm 0.18$ & $0.14 \pm 0.14$ & $0.09 \pm 0.082$ \\
Married (yes) & $1.0 \pm 1.2$ & $1.3 \pm 1.0$ & $-0.057 \pm 0.06$ \\
Tender Joint Counts & $-0.3 \pm 0.3$ & $-0.12 \pm 0.18$ & $0.011 \pm 0.14$ \\
Swollen Joint Counts & $0.5 \pm 0.66$ & $0.32 \pm 0.37$ & $0.2 \pm 0.18$ \\
CRP (mg/dl) & $-0.42 \pm 0.51$ & $-0.26 \pm 0.40$ & $-0.18 \pm 0.26$ \\
Pain VAS & $0.11 \pm 0.035^{* *}$ & $0.12 \pm 0.026 * * *$ & $0.081 \pm 0.013^{* * * *}$ \\
Physician's Global Assessment & $0.028 \pm 0.066$ & $-0.017 \pm 0.045$ & $-0.0089 \pm 0.026$ \\
EQ5D & $0.093 \pm 0.056$ & $0.093 \pm 0.037 *$ & $0.043 \pm 0.018^{*}$ \\
K6 scale(Anxiety/Depression) & $-0.15 \pm 0.14$ & $0.0027 \pm 0.091$ & $0.56 \pm 0.59$ \\
\hline
\end{tabular}

Value are expressed as Estimate \pm Standard Error. ${ }^{*} p<0.05,{ }^{* * *} p<0.0001$

Conclusions: Discrepancy exists between routine in-hospital PtGA and anonymized PtGA.

The high pain VAS scale and low QOL are risk factors that could make the difference between routine PtGA from anonymized PtGA. There is a possibility that high pain VAS score and low QOL influence the reliability of PtGA.

Disclosure of Interest: None declared

DOI: 10.1136/annrheumdis-2017-eular.1488

\section{THU0669 THE ASSOCIATION BETWEEN HARRIS HIP SCORE AND DISEASE ACTIVITY OR HIP MRI FEATURES IN ANKYLOSING SPONDYLITIS}

Z. Wu ${ }^{1}$, L. $\mathrm{He}^{2}$, M. Yang ${ }^{3}$, Y. Liu ${ }^{4}$, D. He ${ }^{5}$, Y. Zhang ${ }^{6}$, C. Wang ${ }^{7}, \mathrm{H} . \mathrm{Xu}^{8}$ ${ }^{1}$ Department of Rheumatology, Xijing Hospital; ${ }^{2}$ Department of Rheumatology, The First Affiliated Hospital of Xi'An JiaoTong University Hospital, Xi'An; ${ }^{3}$ Department of Rheumatology, Nanfang Hospital, Guangzhou; ${ }^{4}$ Department of Rheumatology, West China Hospital, Sichuang; ${ }^{5}$ Department of Rheumatology, Guang Hua Hospital, Shanghai; ${ }^{6}$ Department of Rheumatology, Tangdu Hospital; ${ }^{7}$ Medical Affairs, Xian-Janssen Pharmaceutical Ltd, Xi'An; ${ }^{8}$ Department of Rheumatology and Immunology, Shanghai Changzheng Hospital, Shanghai, China

Background: Hip involvement is a common clinical feature observed in ankylosing spondylitis (AS) patients and it often leads to substantial restriction of the body functions. ${ }^{1}$ Hip involvement in AS is mainly assessed by radiographic changes, clinical symptoms or MRI. The Harris hip score (HHS) is a valuable assessment tool to measure health status of AS patients. However, the relationship between HHS and other clinical indices is unknown.

Objectives: To evaluate relationship between HHS and other commonly used clinical indices.

Methods: In this multicentre, observational study, AS patients with hip clinical manifestation are enrolled and randomly assigned to infliximab treatment (group I, with/without DMARDs and/or NSAIDs) or conventional therapy (group II, DMARDs and/or NSAIDs). Primary endpoint: to compare functional improvement of hip joint (HHS) between two treatment groups (infliximab and conventional therapy) at week 30. Secondary endpoint: to compare disease activity and functional improvement of AS and radiologic progression of hip joint between two treatment groups at week 30 and 52. Association between baseline HHS and disease activity measures such as Bath ankylosing spondylitis (BAS)-functional index (BASFI), BAS disease activity index (BASDAI), AS disease activity score (ASDAS), CRP and ESR was analysed using Pearson correlation coefficient. BAS metrology index (BASMI) and hip imaging functions (MRI of hip, BAS radiology hip index [BASRI-h]) were analysed using independent two-sample t-test or ANOVA based on data characteristics.

Results: Study is ongoing; currently, only baseline information is analyzed. Baseline demographics and disease characteristics did not show any significant difference between groups. Almost all baseline disease activity measures showed significant Pearson correlation (high correlation in BASFI $=0.646$ ) with HHS, except for BASDAI (table 1). Significant association between HHS and three MRI scores (articular cartilage stripping, bone destruction under joint surface, femoral head bone marrow cavity edema) and BASRI-h was shown (table 2). Also, significant correlation was shown between BASMI and HHS ( $F$ value [degree of freedom] $=4.70[4] ; p=0.0022$ ).

Table 1. Association between HHS and disease activity measures at baseline

\begin{tabular}{lccc}
\hline & Mean $(\mathrm{SD})$ & Pearson correlation-HHS & P Value \\
\hline ASDAS & $2.81(0.904)$ & -0.449 & 0.0004 \\
BASDAI & $4.60(1.109)$ & -0.169 & 0.1691 \\
BASFI & $3.79(2.050)$ & -0.646 & $<0.0001$ \\
CRP & $12.270(19.4810)$ & -0.348 & 0.0074 \\
ESR & $22.1(24.06)$ & -0.386 & 0.0023
\end{tabular}

Table 2. Association between HHS and hip imaging functions at baseline

\begin{tabular}{lccccc}
\hline & & $\mathrm{N}$ & $\begin{array}{c}\mathrm{HHS}, \\
\text { mean }(\mathrm{SD})\end{array}$ & $\begin{array}{c}\text { T-test } \\
\text { (degree of freedom) }\end{array}$ & P value \\
\hline Articular cartilage stripping & No & 29 & $62.5(9.13)$ & $2.27(54.97)$ & 0.0271 \\
& Yes & 32 & $55.9(13.39)$ & & \\
Bone destruction under joint surface & No 22 & $63.8(4.66)$ & $3.08(49.29)$ & 0.0034 \\
& Yes & 38 & $56.2(14.02)$ & & \\
Femoral head bone marrow cavity edema & No 29 & $62.4(9.37)$ & $2.18(58.00)$ & 0.0332 \\
& Yes & 31 & $55.8(13.46)$ & & \\
BASRI-h (at least one Hip $\geq 1)$ & - & 29 & $54.7(14.01)$ & $-3.00(37.55)$ & 0.0048 \\
\hline
\end{tabular}

Conclusions: The baseline results of HHS were found to be well associated with disease activity scores like BASFI, ASDAS and BASMI and four hip imaging features in AS patients with hip involvement.

References:

[1] Jeong H, et al. Korean J Intern Med. 2017; 32(1): 158-164.

Disclosure of Interest: Z. Wu: None declared, L. He: None declared, M. Yang: None declared, Y. Liu: None declared, D. He: None declared, Y. Zhang: None declared, C. Wang Employee of: Xi'an Janssen pharmaceuticals Ltd., H. Xu: None declared

DOI: 10.1136/annrheumdis-2017-eular.3724

\section{THU0670 DETECTION OF SERUM ANTI-DNASE I ANTIBODIES IN SYSTEMIC LUPUS ERYTHEMATOSUS USING IMPROVED IMMUNOSORBENT ASSAY}

A.S. Trofimenko ${ }^{1,2}$, I.P. Gontar ${ }^{1}$, I.A. Zborovskaya ${ }^{1}$, L.N. Shilova ${ }^{2}$,

E.G. Korenskaya ${ }^{2} .{ }^{1}$ Research Institute for Clinical and Experimental Rheumatology; ${ }^{2}$ Volgograd State Medical University, Volgograd, Russian Federation

Background: Diagnosis of systemic lupus erythematosus (SLE) is a sophisticated problem in most of the disease cases. The reasons of this include common diagnostic markers of SLE (ANA, anti-dsDNA, anti-Sm), that are insufficiently reliable itself as well. One of possible ways to overcome these difficulties is searching for new SLE markers. These emerging diagnostic tools are not only to be valuable for diagnosis establishment, but also to provide economical efficiency and facility in common use.

Objectives: To compare diagnostic efficiency of anti-DNase I antibodies measured by conventional ELISA and by originally modified enzyme immunosorbent assay using magnetic polyacrylamide beads as an antigen carrier.

Methods: The research was carried out in agreement with the WMA Declaration of Helsinki principles, it was approved by the Regional Committee on Medical Ethics. All the patients signed the informed consent. We have enrolled 54 in-hospital adult patients with SLE, verified by the ACR criteria (1997). Control group $(n=52)$ was comprised of patients with rheumatoid arthritis, systemic sclerosis, systemic vasculitides, dermatomyositis, and Sjogren's disease. Serum 